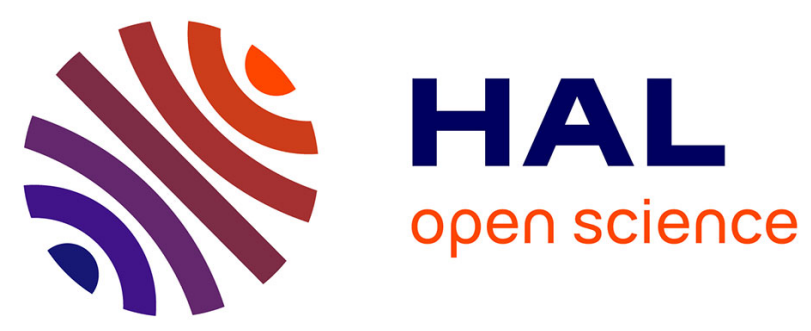

\title{
Output to Input Saturation Transformation: Demonstration and Application to Disturbed Linear Systems
}

\author{
Emmanuel Chambon, Laurent Burlion, Pierre Apkarian
}

\section{- To cite this version:}

Emmanuel Chambon, Laurent Burlion, Pierre Apkarian. Output to Input Saturation Transformation: Demonstration and Application to Disturbed Linear Systems. 54th IEEE Conference on Decision and Control, Dec 2015, Osaka, Japan. hal-01239047

\section{HAL Id: hal-01239047 https://hal.science/hal-01239047}

Submitted on 7 Dec 2015

HAL is a multi-disciplinary open access archive for the deposit and dissemination of scientific research documents, whether they are published or not. The documents may come from teaching and research institutions in France or abroad, or from public or private research centers.
L'archive ouverte pluridisciplinaire HAL, est destinée au dépôt et à la diffusion de documents scientifiques de niveau recherche, publiés ou non, émanant des établissements d'enseignement et de recherche français ou étrangers, des laboratoires publics ou privés. 


\title{
Output to Input Saturation Transformation: Demonstration and Application to Disturbed Linear Systems
}

\author{
Emmanuel Chambon*, Laurent Burlion* and Pierre Apkarian*
}

\begin{abstract}
In the case of linear systems, control law design is often performed so that the resulting closed-loop meets specific frequency requirements. However, in many cases, it may be observed that the obtained controller does not enforce timedomain requirements amongst which the objective of keeping an output variable in a given interval. In this article, a transformation is proposed to convert expected bounds on an output variable into time-varying saturations on the synthesized linear control law. It is demonstrated that the resulting closedloop is stable and satisfies time-domain constraints in the presence of unknown bounded disturbance. An application to a linear ball and beam model is presented.
\end{abstract}

\section{INTRODUCTION}

To stabilise a given system, many techniques exist to obtain a control law satisfying to specified constraints. As far as $M I M O$ systems are concerned, nonsmooth optimization techniques can for example be used to enforce frequencydomain requirements.

However it is possible that, using this control law, timedomain requirements on a so-called regulated variable $\alpha=$ $C_{\alpha} x \in \mathbb{R}$ are not enforced. This is illustrated on Fig. 1 where $\alpha$ time-response violates expected bounds $[\underline{\alpha}(t), \bar{\alpha}(t)]$.

The output- to input-saturation transformation (OIST) theory was first detailed in [1], [2] and proposes to find a remedy to this by reformulating 'saturations' (or expected bounds) on the regulated variable $\alpha$ into saturations on the controller output $u$. Other strategies include [3], [4] which use anti-windup loops to constrain the state or outputs in the time-domain. OIST is illustrated on the bottom of Fig. 1 where an ad hoc saturating block is inserted before the

*Emmanuel Chambon, Laurent Burlion and Pierre Apkarian are with Systems Control and Flight Dynamics Department, Onera - Centre de Toulouse, FR-31000 Toulouse, France, emmanuel. chambondonera. fr
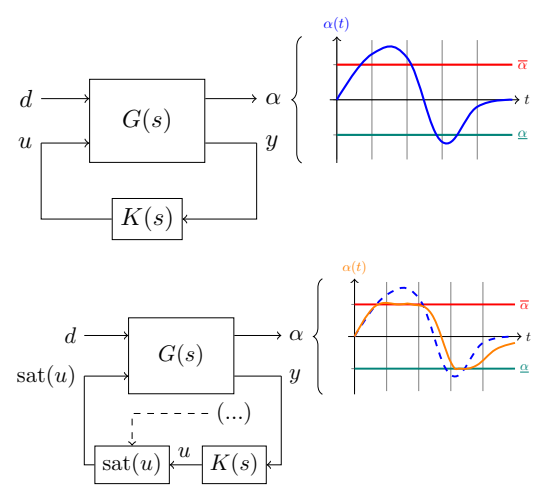

Fig. 1. Top: system $(G)$ in closed-loop with controller K. Bottom: expected performance (in orange) with $O I S T$ in the loop system control input. As illustrated on this figure, additional information may be required to express these saturations which will be demonstrated in the following.

In this paper, it is proposed to extend and demonstrate the theoretical background of the OIST approach in the case of known linear systems with unknown disturbance input and unknown error measurements. The transformation is detailed and a proof of stability making use of existing results on the stability of a class of saturated open-loop stable nonlinear systems [5], [6] is provided. The main addition to the OIST theory proposed in this paper is to formalize timevarying gains to avoid input saturation crossings.

The article is organised as follows: the OIST problem is formalized in Sect. II. Then, the output to input saturation transformation used to solve this problem is presented in Sect. III. Stability of the system in closed-loop with the obtained saturated control is guaranteed and proved in Sect. IV. Finally, the whole approach is applied to a linear ball and beam model in Sect. V and conclusions are drawn in Sect. VI.

\section{THE OIST PROBLEM}

\section{A. Definitions and Notations}

If not stated otherwise, the state-space representation of a linear system $(G)$ is denoted $G=(A, B, C, D)$. The state is then denoted $x \in \mathbb{R}^{n}$, the measurements vector is denoted $y \in \mathbb{R}^{m}$ with $m \leq n$ and the notation $u$ denotes control inputs while $d$ denotes unknown inputs and/or disturbances. Inequalities involving matrices of identical dimensions are understood component-wise. For a given bounded variable $x(t)$, the bounds are denoted $(\underline{x}, \bar{x})$, i.e. $\forall t, \underline{x}(t) \leq x(t) \leq$ $\bar{x}(t)$. The saturation and deadzone functions are respectively denoted $\operatorname{sat}_{x}^{\bar{x}}(x)$ and $\operatorname{Dz}(x)$ and are related to each other by $\operatorname{sat}_{x}^{\bar{x}}(x)=x-\mathrm{Dz}(x)$.

$\bar{D}$ efinition 1: Let $\forall x \in \mathbb{R}, F(x)=x e^{x}$. The inverse function of $F$ is the Lambert function denoted $W_{0}(y)$ which satisfies $\forall y \in \mathbb{R}, F\left(W_{0}(y)\right)=W_{0}(y) e^{W_{0}(y)}=y$.

Definition 2: Let define the constants $\xi:=\frac{1}{2} W_{0}\left(\frac{1}{e}\right)+\frac{1}{2}$ and $\Xi:=\xi-\tanh (\xi) \xi>0$. Using these notations, also define the following functions, $\forall(x, y) \in \mathbb{R}^{2}$ :

$$
\begin{array}{ll}
f_{\text {abs }}(x) & :=\tanh (x) x+\Xi \\
f_{\max }(x, y) & :=\frac{1}{2}[x+y+\tanh (x-y)(x-y)+\Xi] \\
g(x, y) & :=f_{\max }\left(f_{\text {abs }}(x), f_{\text {abs }}(y)\right)
\end{array}
$$

Remark 1: Note $\xi$ is the solution to the equation $(2 x-1) e^{2 x}=1$. Also note that $f_{\text {abs }}, f_{\max }$ and $g$ are continuous differentiable over $\mathbb{R}$ or $\mathbb{R}^{2}$. Moreover, 
- $\forall x \in \mathbb{R}, f_{\text {abs }}(x) \geq|x|$

- $\forall(x, y) \in \mathbb{R}^{2}, f_{\max }(x, y) \geq \max (x, y)$.

Proof: straightforward using function analysis basics.

Definition 3: Let $k \in \mathbb{N}$. Considering a linear system $(G)$, the regulated variable $\alpha=C_{\alpha} x$ is said to be of relative degree $k$ with respect to $u$ if and only if

$$
\forall i<k-1, C_{\alpha} A^{i} B=0 \text { and } C_{\alpha} A^{k-1} B \neq 0
$$

Definition 4: Let $S=\left[\begin{array}{lll}s_{0} & \ldots & s_{k}\end{array}\right] \in \mathbb{R}^{k+1}$. The function

$$
\sigma(S)=\left[\begin{array}{lll}
\sigma\left(s_{0}\right) & \ldots & \sigma\left(s_{k}\right)
\end{array}\right]
$$

where

$$
\sigma\left(s_{i}\right):=\left\{\begin{array}{lll}
s_{i-1} & \text { if } \quad 1 \leq i \leq k \\
s_{k} & \text { if } \quad i=0
\end{array}\right.
$$

is called the cyclic permutation of length $k+1$ on the elements of $S$.

\section{B. Problem formulation}

Let consider a linear system $(G)$ with the following statespace representation:

$$
(G)\left\{\begin{aligned}
\dot{x} & =A x+B_{u} u+B_{d} d \\
y & =x
\end{aligned}\right.
$$

where $u$ is the control input and $d$ an unknown disturbance. It is supposed a stabilizing control law $u=K(s) y$ has been designed for this system. The corresponding OIST problem is formalized into:

Problem 1: Find $[\underline{u}(t), \bar{u}(t)]$ and $\mathcal{C}_{0}$ such that

$$
\alpha(t) \in[\underline{\alpha}(t), \bar{\alpha}(t)], \forall t
$$

for the system described as

$$
\left\{\begin{array}{l}
\dot{x}=A x+B_{u} u+B_{d} d \\
y=x \\
\alpha=C_{\alpha} x+E_{\alpha} e \\
u \in \underline{u}(t), \bar{u}(t)] \\
x_{0} \in \mathcal{C}_{0}
\end{array}\right.
$$

where $x \in \mathbb{R}^{n}, y \in \mathbb{R}^{m}, u \in \mathbb{R}, d \in \mathbb{R}$ is an unknown bounded disturbance and $e \in \mathbb{R}^{n}$ is an unknown bounded signal with $E_{\alpha} \in \mathbb{R}^{1 \times n}$.

\section{Hypotheses}

All the hypotheses satisfied by the systems considered in this article are presented here. Notations are those of $\mathrm{Pb} .1$.

Hypothesis 1: Let $(k, l) \in \mathbb{N}^{2}$ such that $1 \leq l \leq k$. It is supposed the regulated variable $\alpha$ is of relative degree $k$ (resp. $l$ ) wrt. $u$ (resp. $d$ ).

Let $\mathcal{D}(t)=\left[\begin{array}{llll}d & \dot{d} & \ldots & d^{(k-l-1)}\end{array}\right]^{\top} \in \mathbb{R}^{k-l}$ and $\mathcal{E}(t)=$ $\left[\begin{array}{llll}e & \dot{e} & \ldots & e^{(k)}\end{array}\right]^{\top} \in \mathbb{R}^{(k+1) \times n}$.

Hypothesis 2: Time-varying bounds $[\underline{\mathcal{D}}(t), \overline{\mathcal{D}}(t)]$ on the unknown disturbance and its derivatives $\mathcal{D}(t)$ are supposed to be known, that is

$$
\forall t, \underline{\mathcal{D}}(t) \leq \mathcal{D}(t) \leq \overline{\mathcal{D}}(t)
$$

or, more precisely

$$
\forall t, \forall i \text { s.t. } 0 \leq i \leq k-l-1, \underline{d^{(i)}}(t) \leq d^{(i)}(t) \leq \overline{d^{(i)}}(t)
$$

Moreover, $d$ is supposed to be of finite energy $\|d\|_{2}$. The same holds for $\mathcal{E}(t)$ with time-varying bounds $[\underline{\mathcal{E}}(t), \overline{\mathcal{E}}(t)]$ and finite energy $\|e\|_{2}$.

Hypothesis 3: The transfer function $T_{u \rightarrow \alpha}(s)$ from input $u$ to regulated variable $\alpha$ only has strictly stable zeros.

Hypothesis 4: Using notations in Hyp. 1, the following is satisfied: $2 l>k$.

\section{OIST TRANSFORMATION}

The output- to input-saturation transformation is presented in this section in the case of known linear systems satisfying to Hyp. 1 to 4 . In presence of unknown disturbances $d$ and $e$, the main idea is to use time-varying coefficients in the obtained saturations so as to avoid crossings.

\section{A. From the regulated variable to the input}

Under Hyp. 1 and 4, the $k$-th derivative of the regulated variable $\alpha$ in function of $u, d$ and $e^{(k)}$ has the following expression

$\alpha^{(k)}=C_{\alpha} A^{k} x+E_{\alpha} e^{(k)}+C_{\alpha} A^{k-1} B_{u} u+\sum_{j=l}^{k} C_{\alpha} A^{j-1} B_{d} d^{(k-j)}$

The derivative of order $k$ is the first derivative of $\alpha$ to depend on $u$. By derivating $k$ times the variable $\alpha$ and expected bounds $[\underline{\alpha}(t), \bar{\alpha}(t)]$, one can thus express saturations on $u$.

\section{B. Keeping a variable in an interval}

Let $\kappa(t)=\left[\begin{array}{lll}\kappa_{1}(t) & \ldots & \kappa_{k}(t)\end{array}\right] \in \mathbb{R}_{+}^{k}$ a vector of known positive time-varying signals, $\underline{\alpha^{(0)}}=\underline{\alpha}$ and $\overline{\alpha^{(0)}}=\bar{\alpha}$. Also consider known vectors $\mathcal{A}(t)=\left[\begin{array}{lll}\alpha & \ldots & \alpha^{(k)}\end{array}\right]$ and $\underline{\Omega}(t)=$ $\left[\begin{array}{lll}\underline{\alpha^{(0)}} & \cdots & \underline{\alpha^{(k)}}\end{array}\right]^{1}$.

Lemma 1: Let $\mathcal{A}(0) \in[\underline{\Omega}(0), \bar{\Omega}(0)]$ and $\forall j, 1 \leq j \leq$ $k, \forall t$ :

$$
\begin{aligned}
& \underline{\alpha^{(j)}}(t)=\kappa_{j}(t)\left(\underline{\alpha^{(j-1)}}-\alpha^{(j-1)}\right)+\overparen{\overparen{\alpha^{(j-1)}}} \\
& \overline{\alpha^{(j)}}(t)=\kappa_{j}(t)\left(\overline{\alpha^{(j-1)}}-\alpha^{(j-1)}\right)+\overparen{\alpha^{(j-1)}}
\end{aligned}
$$

Then,

$$
\begin{aligned}
& \alpha^{(k)} \in\left[\underline{\alpha^{(k)}}(t), \overline{\alpha^{(k)}}(t)\right], \forall t \Rightarrow \alpha \in[\underline{\alpha}(t), \bar{\alpha}(t)], \forall t \\
& \text { Proof: } \\
& \text { straightforward using proof by contradiction. }
\end{aligned}
$$

\section{Control saturations}

Under Hyp. 1, it is ensured $C_{\alpha} A^{k-1} B_{u} \neq 0$. Let also suppose that $C_{\alpha} A^{k-1} B_{u}>0$. In case this is not satisfied, this means the definition of $\underline{u}$ and $\bar{u}$ were exchanged. Without changing these definitions, the saturated value of the command $u$ is then obtained using

$$
\operatorname{sat}(u)=\max (\min (\underline{u}, \bar{u}), \min (u, \max (\underline{u}, \bar{u})))
$$

Proof:

- Suppose $\underline{u}>\bar{u}$. Then, $\min (\underline{u}, \bar{u})=\bar{u}$ and $\max (\underline{u}, \bar{u})=$ $\underline{u}$. Eq. (12) becomes sat $(u)=\max (\bar{u}, \min (u, \underline{u}))$.

$1 \bar{\Omega}(t)$ is defined in a similar way. 
- Suppose $\underline{u}<\bar{u}$. Then, $\min (\underline{u}, \bar{u})=\underline{u}$ and $\max (\underline{u}, \bar{u})=$ $\bar{u}$. Eq. (12) becomes $\operatorname{sat}(u)=\max (\underline{u}, \min (u, \bar{u}))$.

In both cases, the expected result is obtained.

Considering Lemma 1 and Eq. (9), and supposing the expression of $\alpha^{(k)}(t)$ is known, the lower bound from Eq. (13) on Fig. 2 is obtained (to obtain the upper bound, a similar approach is used). With a slight abuse of notation, the (more conservative) second expression will be used.

Remark 2: For this definition to be consistent and to avoid control saturations crossings, one must ensure that

$$
\begin{aligned}
\overline{\alpha^{(k)}}(t)- & \underline{\alpha^{(k)}}(t) \geq 2\left|E_{\alpha}\right| \max \left(\left|\underline{e^{(k)}}\right|,\left|\overline{e^{(k)}}\right|\right) \\
& +2 \sum_{j=l}^{k}\left|C_{\alpha} A^{j-1} B_{d}\right| \max \left(\left|\underline{d^{(k-j)}}\right|,\left|\overline{d^{(k-j)}}\right|\right)
\end{aligned}
$$

\section{Determining $\alpha^{(j)}$ and $\overline{\alpha^{(j)}}$ expressions}

The expressions of the bounds on the successive derivatives of the regulated variable $\alpha$ can be determined using the iterative definition in Eq. (10). For the sake of space, only the lower bounds are considered. Determining the upper bounds is then straightforward. For any integer $j$ such that $1 \leq j \leq k$, let consider the two following vectors:

- $U^{j}(t)=\left[\begin{array}{lll}u_{0}^{j}(t) & \ldots & u_{k}^{j}(t)\end{array}\right]$ where $u_{j}^{j}(t)=1$ and $\forall i>j, u_{i}^{j}(t)=0$;

- $V^{j}(t)=\left[\begin{array}{llll}v_{0}^{j}(t) & \ldots & v_{k-l-1}^{j}(t)\end{array}\right]$ where: $\forall i \quad>$ $\max (-1, j-l-1), v_{i}^{j}(t)=0$ and $v_{0}^{l+1}(t)=$ $u_{l}^{l+1}(t) C_{\alpha} A^{l-1} B_{d}$,

Let $\underline{\mathcal{A}}=\left[\begin{array}{llll}\underline{\alpha} & \stackrel{\dot{\alpha}}{\underline{\alpha}} \ldots & (\underline{\alpha})^{(k)}\end{array}\right]^{\top}$ and $\Theta=$ $\left[\begin{array}{llll}C_{\alpha} & C_{\alpha} A & \ldots & C_{\alpha} A^{k}\end{array}\right]^{\top}$. Using these definitions and supposing $\mathcal{E}(t)$ and $\mathcal{D}(t)$ are known, one can express the lower bound $\alpha^{(j)}(t)$ to be satisfied by $\alpha^{(j)}(t)$ as:

$$
\begin{aligned}
\underline{\alpha^{(j)}}(t)=U^{j}(t)\{\underline{\mathcal{A}}-\Theta x- & \left.\mathcal{E}(t) E_{\alpha}^{\top}\right\}+C_{\alpha} A^{j} x \\
& +E_{\alpha} e^{(j)}-V^{j}(t) \mathcal{D}(t) \in \mathbb{R}
\end{aligned}
$$

Then, using Eq. (10), the formulas in Eq. (16) on Fig. 3 are obtained where $\sigma\left(U^{j-1}(t)\right)$ is the cyclic permutation of length $k+1$ on the elements of $U^{j-1}(t)$.

Proof: tedious rewriting of Eq. (15) in explicit form and using Eq. (10) to express $\underline{\alpha^{(j+1)}}$ for $j \geq 0$ starting with $\underline{\alpha^{(0)}}(t)=\underline{\alpha}(t)$ leads to Eq. (16).

Remark 3: Using the previous iterative expressions and the fact that $u_{0}^{1}(t)=\kappa_{1}(t)$, one can easily determine that $\forall j, 0 \leq j \leq k, u_{j}^{j+1}(t)=\sum_{w=1}^{j+1} \kappa_{w}(t)$.

In case $\mathcal{E}(t)$ and $\mathcal{D}(t)$ are not known and using notations in Def. 2, the differentiable (yet more conservative) expression in Eq. (17) on Fig. 4 is obtained. With a slight abuse of notation, it is chosen as the new definition of $\underline{\alpha^{(j)}}(t)$.

\section{E. Avoiding crossings}

Let define $\forall j, 1 \leq j \leq k, \Omega_{j}(t):=\overline{\alpha^{(j)}}(t)-\underline{\alpha^{(j)}}(t)$. Crossings of the saturations imposed to $u$ (and the successive derivatives of $\alpha$ ) are avoided if some properties are satisfied by those terms. In the following, it is proposed to identify these properties from which Th. 1 is obtained.
1) In case $\forall j$ s.t. $1 \leq j<l$ : the following lemma is considered

Lemma 2: Supposing $\forall t, \kappa_{j}(t)>0, \forall j, 1 \leq j<l$,

$\Omega_{j}(t)=\kappa_{j}(t)\left[\Omega_{j-1}(t)-2\left|E_{\alpha}\right| g\left(\underline{e^{(j-1)}}, \overline{e^{(j-1)}}\right)\right]+\dot{\Omega}_{j-1}(t)$

Proof: by definition,

$$
\begin{aligned}
\Omega_{j}(t)= & \overline{\alpha^{(j)}}(t)-\underline{\alpha^{(j)}}(t) \\
= & \kappa_{j}(t)\left(\overline{\alpha^{(j-1)}}(t)-\alpha^{(j-1)}(t)\right)+\overparen{\alpha^{(j-1)}}(t) \\
& -\kappa_{j}(t)\left(\underline{\alpha^{(j-1)}}(t)-\alpha^{(j-1)}(t)\right)-\stackrel{\overbrace{}^{(j-1)}}{\cdot}(t)
\end{aligned}
$$

where $\alpha^{(j-1)}=C_{\alpha} A^{j-1} x+E_{\alpha} e^{(j-1)}$. Since $e$ and its derivatives are not known, it is not possible to simplify $\alpha^{(j-1)}(t)$ in the expression of $\Omega_{j}(t)$. However, under Hyp. 2 and supposing $\kappa_{j}(t)>0$, the conservative expression in Eq. (18) is obtained.

2) In case $\forall j$ s.t. $l \leq j \leq k$ : in the same vein

Lemma 3: Supposing $\forall t, \kappa_{j}(t)>0, \forall j, l \leq j \leq k$,

$$
\begin{aligned}
& \Omega_{j}(t)=\kappa_{j}(t)\left[\Omega_{j-1}(t)-2\left|E_{\alpha}\right| g\left(\underline{e^{(j-1)}}, \overline{e^{(j-1)}}\right)\right. \\
& \left.-2 \sum_{w=l}^{j-1}\left|C_{\alpha} A^{w-1} B_{d}\right| g\left(\underline{d^{(j-w-1)}}, \overline{d^{(j-w-1)}}\right)\right]+\dot{\Omega}_{j-1}(t)
\end{aligned}
$$

Proof: Similar to previous proof where $j \geq l$ implies a different expression for $\alpha^{(j-1)}(t)$.

3) Theorem: the following theorem is proposed

Theorem 1: Saturations crossings are avoided if

- $\bar{\alpha}(t)$ and $\underline{\alpha}(t)$ are chosen such that

$$
\forall t, \Omega_{0}(t)=\bar{\alpha}(t)-\underline{\alpha}(t)>2\left|E_{\alpha}\right| g(\underline{e}(t), \bar{e}(t))
$$

- $\forall j, 1 \leq j<l$, one ensures

$$
\Omega_{j}(t)>2\left|E_{\alpha}\right| g\left(\underline{e^{(j)}}(t), \overline{e^{(j)}}(t)\right)
$$

by choosing

$$
\kappa_{j}(t):=\frac{\breve{\kappa}_{j}-\dot{\Omega}_{j-1}(t)+2\left|E_{\alpha}\right| g\left(\underline{e^{(j)}}(t), \overline{e^{(j)}}(t)\right)}{\Omega_{j-1}(t)-2\left|E_{\alpha}\right| g\left(\underline{e^{(j-1)}}(t), \overline{e^{(j-1)}}(t)\right)}
$$

where the constant $\breve{\kappa}_{j}$ satisfies

$$
\forall t, \breve{\kappa}_{j}>\dot{\Omega}_{j-1}(t)-2\left|E_{\alpha}\right| g\left(\underline{e^{(j)}}(t), \overline{e^{(j)}}(t)\right)
$$

Note that the denominator in Eq. (23) is strictly positive by definition of $\kappa_{j-1}(t)$ and of $\Omega_{0}(t)$ in case $j=1$.

- $\forall j, l \leq j \leq k$, one ensures

$$
\begin{aligned}
& \Omega_{j}(t)>2\left|E_{\alpha}\right| g\left(\underline{e^{(j)}}(t), \overline{e^{(j)}}(t)\right) \\
& \quad+2 \sum_{w=l}^{j}\left|C_{\alpha} A^{w-1} B_{d}\right| g\left(\underline{d^{(j-w)}}(t), \overline{d^{(j-w)}}(t)\right)
\end{aligned}
$$

by choosing $\kappa_{j}(t)$ as in Eq. (26) on Fig. 5 where the constant $\breve{\kappa}_{j}$ satisfies

$$
\begin{aligned}
\forall t, \breve{\kappa}_{j}>\dot{\Omega}_{j-1}(t)-2\left|E_{\alpha}\right| g\left(\underline{e^{(j)}}(t), \overline{e^{(j)}}(t)\right) \\
-2 \sum_{w=l}^{j}\left|C_{\alpha} A^{w-1} B_{d}\right| g\left(\underline{d^{(j-w)}}(t), \overline{d^{(j-w)}}(t)\right)
\end{aligned}
$$




$$
\begin{aligned}
\underline{u}(t) & =\frac{1}{C_{\alpha} A^{k-1} B_{u}}\left[\underline{\alpha^{(k)}}(t)-C_{\alpha} A^{k} x+\left|E_{\alpha}\right| \max \left(\left|\underline{e^{(k)}}\right|,\left|\overline{e^{(k)}}\right|\right)+\left|\sum_{j=l}^{k} C_{\alpha} A^{j-1} B_{d} d^{(k-j)}\right|\right] \\
& \leq \frac{1}{C_{\alpha} A^{k-1} B_{u}}\left[\underline{\alpha^{(k)}}(t)-C_{\alpha} A^{k} x+\left|E_{\alpha}\right| \max \left(\left|\underline{\mid e^{(k)}}\right|,\left|\overline{e^{(k)}}\right|\right)+\sum_{j=l}^{k}\left|C_{\alpha} A^{j-1} B_{d}\right| \max \left(\left|\underline{d^{(k-j)}}\right|,\left|\overline{d^{(k-j)}}\right|\right)\right]
\end{aligned}
$$

Fig. 2. Lower control saturation expression

$$
\begin{array}{rlrl} 
& U^{0}(t)= & {\left[\begin{array}{llll}
1 & 0 & \ldots & 0
\end{array}\right]} \\
\forall j \text { s.t. } 1 \leq j \leq k, & U^{j}(t)= & \kappa_{j}(t) U^{j-1}(t)+\dot{U}^{j-1}(t)+\sigma\left(U^{j-1}(t)\right) \\
\forall j \text { s.t. } 0 \leq j \leq l, & V^{j}(t)= & 0 & \\
\forall j \text { s.t. } l<j \leq k-l, & V^{j}(t) & = & \kappa_{j}(t)\left(V^{j-1}(t)+C_{\alpha} A^{j-l-1}\left[\begin{array}{llll}
A^{l-1} B_{d} & \ldots & A^{2 l-k} B_{d}
\end{array}\right]\right)+\dot{V}^{j-1}(t) \\
& & & \quad+\sigma\left(V^{j-1}(t)\right)+\left[\begin{array}{lllll}
\sum_{w=0}^{j-l-1} u_{l-1+w}^{j-1}(t) C_{\alpha} A^{l-1+w} B_{d} & 0 & \ldots & 0
\end{array}\right]
\end{array}
$$

Fig. 3. Iterative definitions of the coefficients in $\underline{\alpha^{(j)}}$

$$
\begin{aligned}
& \underline{\alpha^{(j)}}(t)=\kappa_{j}(t)\left[U^{j-1}(t)\{\underline{\mathcal{A}}-\Theta x\}+f_{\text {abs }}\left(U^{j-1}(t)\right) g(\underline{\mathcal{E}}(t), \overline{\mathcal{E}}(t))\left|E_{\alpha}^{\top}\right|\right. \\
& \left.+f_{\text {abs }}\left(V^{j-1}(t)+C_{\alpha} A^{j-l-1}\left[\begin{array}{lll}
A^{l-1} B_{d} & \ldots & A^{2 l-k} B_{d}
\end{array}\right]\right) g(\underline{\mathcal{D}}(t), \overline{\mathcal{D}}(t))\right]+C_{\alpha} A^{j} x+\left|E_{\alpha}\right| g\left(\underline{e^{(j)}}, \overline{e^{(j)}}\right) \\
& +\left[\dot{U}^{j-1}(t)+\sigma\left(U^{j-1}(t)\right)\right]\{\underline{\mathcal{A}}-\Theta x\}+f_{\text {abs }}\left(\dot{U}^{j-1}(t)+\sigma\left(U^{j-1}(t)\right)\right) g(\underline{\mathcal{E}}(t), \overline{\mathcal{E}}(t))\left|E_{\alpha}^{\top}\right| \\
& +f_{\mathrm{abs}}\left(\dot{V}^{j-1}(t)+\sigma\left(V^{j-1}(t)\right)+\left[\begin{array}{lllll}
\sum_{w=0}^{j-l-1} u_{l-1+w}^{j-1}(t) C_{\alpha} A^{l-1+w} B_{d} & 0 & \ldots & 0
\end{array}\right]\right) g(\underline{\mathcal{D}}(t), \overline{\mathcal{D}}(t))
\end{aligned}
$$

Fig. 4. Explicit expression for $\underline{\alpha}^{(j)}$ where coefficient $\kappa_{j}(t)$ is factorized. Note this is differentiable with respect to $t$

$$
\kappa_{j}(t):=\frac{\breve{\kappa}_{j}-\dot{\Omega}_{j-1}(t)+2\left|E_{\alpha}\right| g\left(\underline{e^{(j)}}(t), \overline{e^{(j)}}(t)\right)+2 \sum_{w=l}^{j}\left|C_{\alpha} A^{w-1} B_{d}\right| g\left(\underline{d^{(j-w)}}(t), \overline{d^{(j-w)}}(t)\right)}{\Omega_{j-1}(t)-2\left|E_{\alpha}\right| g\left(\underline{e^{(j-1)}}, \overline{e^{(j-1)}}\right)-2 \sum_{w=l}^{j-1}\left|C_{\alpha} A^{w-1} B_{d}\right| g\left(\underline{d^{(j-w-1)}}, \overline{d^{(j-w-1)}}\right)}
$$

Fig. 5. Expression of $\kappa_{j}(t)$ for $l \leq j \leq k$

Note that the denominator in Eq. (26) is strictly positive by definition of the previous coefficient $\kappa_{j-1}(t)$.

Proof: straightforward using the results from Sect. IIIE.1 and III-E.2.

\section{CLOSED-LOOP STABILITY}

In this section, the stability of the system in closed-loop with the dynamic controller $u=K(s) y=K(s) x$ and the proposed time-varying saturations $(\underline{u}, \bar{u})$ is proved.

\section{A. Considered system}

Replacing $\underline{\alpha^{(k)}}(t)$ in Eq. (13) (Fig. 2) by its expression in Eq. (15) and factorizing by the state vector $x$, the following can be defined

$$
K_{\text {oist }}(t)=\frac{U^{k}(t) \Theta}{C_{\alpha} A^{k-1} B_{u}} \in \mathbb{R}^{1 \times n}
$$

Let $\underline{v}=\underline{u}+K_{\text {oist }}(t) x$ and $\bar{v}=\bar{u}+K_{\text {oist }}(t) x$. By definition of these bounds, see Eq. (13), the following is straightforward

$$
\operatorname{sat}_{\underline{u}}^{\bar{u}}(u)=\operatorname{sat}_{\underline{v}}^{\bar{v}}(v)-K_{\text {oist }}(t) x
$$

In closed-loop with the dynamic controller $K(s)$ and the saturations, the studied system is equivalent to

$$
\left\{\begin{aligned}
\dot{x} & =\left[A-B_{u} K_{\text {oist }}(t)\right] x+B_{u} \operatorname{sat}_{\underline{v}}^{\bar{v}}(v)+B_{d} d \\
y & =x \\
\alpha & =C_{\alpha} x+E_{\alpha} e
\end{aligned}\right.
$$

where $v=u+K_{\text {oist }}(t) x=C_{K} x_{K}+D_{K} y+K_{\text {oist }}(t) x$. The interest of such a reformulation is to obtain a system where the saturations $(\underline{v}, \bar{v})$ do not depend on the state $x$. The following Lemma is considered

Lemma 4: $\forall x \in \mathbb{R}^{n}$, the function $-K_{\text {oist }}(t) x$ is Lipschitz.

Proof: Let define $\forall x \in \mathbb{R}^{n}, f(x)=-K_{\text {oist }}(t) x$. Then $\forall x \in \mathbb{R}^{n}, \frac{\partial f}{\partial x}(x)=-K_{\text {oist }}(t)$. Since $K_{\text {oist }}(t)$ is continuous by definition of the coefficients $\kappa(t)$ and continuity of $[\underline{\mathcal{E}}, \overline{\mathcal{E}}, \underline{\mathcal{D}}, \overline{\mathcal{D}}]$, the function $f$ is continuously differentiable. This implies that $K_{\text {oist }}(t) x$ is a Lipschitz continuous function on $\mathbb{R}^{n}$.

\section{B. Anti-windup design and stability proof}

Due to the presence of a dynamic controller and saturations, unexpected closed-loop behaviour is expected. Antiwindup techniques have been widely studied and used to hinder such behaviour as in [7], [8], [9]. The approach presented in [5], [6] deals with a specific class of nonlinear systems to which the system presented in Eq. (30) belongs. The results used from this work are presented and demonstrated here.

Considering system (30), the following anti-windup with state $x_{a} \in \mathbb{R}^{n}$ is introduced

$$
\left\{\begin{aligned}
\dot{x}_{a} & =\left[A-B_{u} K_{\mathrm{oist}}(t)\right] x_{a}-B_{u} \mathrm{Dz}(v) \\
\theta_{1} & =-K_{\mathrm{oist}}(t) x_{a} \\
\theta_{2} & =x_{a}
\end{aligned}\right.
$$


The control $v$ is modified into

$$
v=K(s)\left(y-\theta_{2}\right)+K_{\text {oist }}(t) x+\theta_{1}
$$

Let consider the following theorem which will be necessary to prove Th. 3 .

Theorem 2: The open-loop system

$$
\dot{x}=\left[A-B_{u} K_{\text {oist }}(t)\right] x
$$

is globally exponentially stable.

Proof: due to space restrictions, only a sketch of the proof is given here. Considering Eq. (29) and (30), this problem is equivalent to studying the stability of system $\dot{x}=A x+B_{u} \underline{u}$. The transfer function between $u$ and the regulated variable $\alpha$ can be written in the following statespace form:

$$
\overbrace{\left[\begin{array}{l}
\Gamma \\
Z
\end{array}\right]}^{\dot{a}}=\left[\begin{array}{cc}
A_{\Gamma} & 0 \\
A_{Z \Gamma} & A_{Z}
\end{array}\right]\left[\begin{array}{l}
\Gamma \\
Z
\end{array}\right]
$$

where $A_{\Gamma}$ describes a chain of integrators [10, Chapter 4] and $A_{Z}$ describes the zero-dynamics. Considering Hyp. 3, $A_{Z}$ eigenvalues are strictly negative. As far as the dynamics of Eq. (34) is concerned, the following candidate Lyapunov positive definite function is considered

$$
V(\Gamma, Z)=\frac{1}{2} \Gamma^{\top} \Gamma+\frac{\epsilon}{2} Z^{\top} Z
$$

where $\epsilon$ is a positive constant. Choosing $\epsilon$ wisely and using the common analysis, this candidate function validates as a Lyapunov function. Even more, global exponential stability of $\dot{x}=\left[A-B_{u} K_{\text {oist }}\right] x$ can be shown.

Before any further development, let consider the following hypothesis which ensures that the origin of the system is reachable with $u=v=0$.

Hypothesis 5: $0 \in[\underline{v}(t), \bar{v}(t)], \forall t$

Since $\underline{v}$ and $\bar{v}$ only depend on known model and design parameters, this hypothesis can be checked prior to any simulation. The core of this article lies in the following theorem which proves stability of the system in closed-loop with the saturated initial controller. Both this theorem and its proof are fully inspired from [5].

Theorem 3: Under Hyp. 1 to 5, the origin of the closedloop system consisting of the system (30), the control law (32) and the anti-windup compensator (31) is globally asymptotically stable.

Proof: the details of the proof of [5, Theorem 1, p. 5436] are applied to our case. Using Th. 2, the Eq. [5, (22) to (27), p. 5437] are valid hence the conclusion of the proof. Note that in our case, the deadzone function $\operatorname{Dz}(v)$ depends on time-varying bounds $(\underline{v}, \bar{v})$. However, the inequality $\|\mathrm{Dz}(v)\| \leq\|v\|$ still stands from Hyp. 5 .

\section{EXAMPLE}

To illustrate the theoretical results presented in this article, the OIST approach is applied to the dynamic control of a ball and beam linear model. The objective is to maintain the ball on the beam even in presence of unknown disturbances.

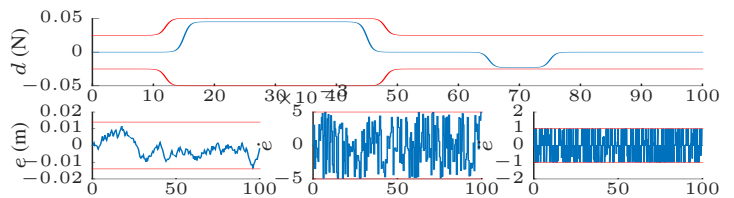

Fig. 6. disturbances $d$ and $e$ along with their known bounds (in red)

\section{A. Model description}

The system is considered linear in the case of small beam inclinations. The beam is actuated using a lever arm. The position of the ball is denoted $r$ and the actuation wheel angle is denoted $\theta$. An unknown disturbance force $d$ is eventually applied to the ball acceleration and an unknown bias $e$ is introduced on the measure of $r$. The state of the system is given by $x=\left[\begin{array}{ll}r & \dot{r}\end{array}\right]^{\top}$. The regulated variable is defined as $\alpha=\left[\begin{array}{ll}1 & 0\end{array}\right] y=r+e$. The beam measuring only $L=$ $1 \mathrm{~m}$, the objective is to satisfy $\forall t, 0.1 \leq \alpha(t) \leq 0.9$ (in meters) while driving the system from $r_{0}=0.5 \mathrm{~m}$ to the setpoint $r_{s}=0.6 \mathrm{~m}$. Considering this regulated variable, it is observed that $l=2$ and $k=2$. The system state-space representation is given by:

$$
A=\left[\begin{array}{ll}
0 & 1 \\
0 & 0
\end{array}\right], B=\left[\begin{array}{rrr}
0 & 0 & 0 \\
1 & -0.21 & 0
\end{array}\right], C=I_{2}, D=\left[\begin{array}{lll}
0 & 0 & 1 \\
0 & 0 & 0
\end{array}\right]
$$

where the inputs are respectively, $d, u$ and $e$. As far as the control design is concerned, $L Q R$ design is used on the adequate augmented system with $R=\operatorname{diag}\left(\left[\begin{array}{ll}10 & 4\end{array}\right]\right)$ and $Q=\operatorname{diag}\left(\left[\begin{array}{lll}1 & 10 & 10\end{array}\right]\right)$. The following state-feedback gain $K_{p}$ with integral-action gain $K_{i}$ are obtained:

$$
K_{p}=\left[\begin{array}{rr}
-0.3156 & -2.0937 \\
0.0322 & -0.7661
\end{array}\right], K_{i}=\left[\begin{array}{l}
0.0644 \\
1.5779
\end{array}\right]
$$

This controller yields good results on $r_{s}$-setpoint tracking. Violation of the expected time-domain performance $0.1 \leq \alpha(t) \leq 0.9$ is however witnessed in presence of the disturbance $d$. This results in the ball falling from the beam. Introducing OIST in the loop, it is expected to comply with this constraint. Note that a more precise selection of the gains $K_{p}$ and $K_{i}$ may deliver more satisfying results. For the purpose of illustrating OIST technique only, better gains are however not sought for.

\section{B. Hypotheses}

- Hyp. 1 and 4 are satisfied: with $\alpha=r, k=l=2$;

- Hyp. 5 is satisfied;

- As far as the unknown disturbances $d$ and $e$ are concerned, their bounds are known as well as the bounds on their required derivatives. The signals on Fig. 6 are used for simulation where the known bounds are shown. Note the abscissa for $e$ is in meters which means an uncertainty on the position measurement of around $1 \mathrm{~cm}$ at maximum. Hyp. 2 is satisfied;

- $T_{u \rightarrow \alpha}(s)=-\frac{0.21}{s^{2}}$ hence Hyp. 3 is satisfied. 


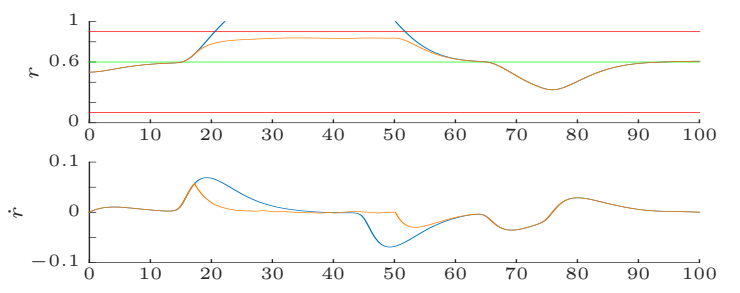

Fig. 7. in orange (resp. blue), system state with (resp. without) OIST in the loop

\section{OIST implementation}

Using results in Sect. III and considering $\underline{\alpha}=0.1 \mathrm{~m}$, $\bar{\alpha}=0.9 \mathrm{~m}$, the following expressions are obtained for the successive $\Omega_{i}$ 's

$$
\begin{aligned}
\Omega_{0}=0.8, \Omega_{1}=\kappa_{1}\left[\Omega_{0}-2 g(\underline{e}, \bar{e})\right], \\
\Omega_{2}=\kappa_{2}\left[\Omega_{1}-2 g\left(\underline{e^{(1)}}, \overline{e^{(1)}}\right)\right]+\dot{\Omega}_{1}
\end{aligned}
$$

where $\Omega_{0}>2 g(\underline{e}, \bar{e})$. Then the values of the $\kappa_{i}$ 's are deduced

$$
\begin{aligned}
\kappa_{1} & =\frac{\breve{\kappa_{1}}+2 g\left(\underline{e^{(1)}}, \overline{e^{(1)}}\right)}{\Omega_{0}-2 g(\underline{e}, \bar{e})} \\
\kappa_{2} & =\frac{\breve{\kappa_{2}}-\dot{\Omega}_{1}+2 g\left(\underline{e^{(2)}}, \overline{e^{(2)}}\right)+2\left|C_{\alpha} A B_{d}\right| \max (|\underline{d}|,|\bar{d}|)}{\Omega_{1}-2 g\left(\underline{e^{(1)}}, \overline{e^{(1)}}\right)}
\end{aligned}
$$

where $\breve{\kappa}=\left[\begin{array}{ll}\breve{\kappa}_{1} & \breve{\kappa}_{2}\end{array}\right]=\left[\begin{array}{ll}30 & 10\end{array}\right]$. Finally, the expressions of the saturations to apply to the controller output are inspired from Eq. (13) on Fig. 2. Note that $C_{\alpha} A B_{u}<0$ in this example so the operator in Eq. (12) is used. As far as the anti-windup design is concerned, the time-varying coefficient $K_{\text {oist }}(t)$ is defined as follows

$$
K_{\text {oist }}(t)=\frac{U^{2}(t) \Theta}{C_{\alpha} A B_{u}}
$$

where $U^{2}(t)=\left[\begin{array}{lll}\kappa_{2} \kappa_{1}+\dot{\kappa_{1}} & \kappa_{1}+\kappa_{2} & 1\end{array}\right]$ and $\Theta=$ $\left[\begin{array}{lll}C_{\alpha} & C_{\alpha} A & C_{\alpha} A^{2}\end{array}\right]^{\top}$.

\section{Simulations and results}

Using results in Sect. V-C, simulations are performed over $100 \mathrm{~s}$. The disturbances $d$ and $e$ shown on Fig. 6 are applied to the system. The disturbance $d$ at time $t=70 \mathrm{~s}$ is used to confirm that when constraints are not violated then the control is not modified. Simulation results are shown on Fig. 7 and 8. The system data are represented in blue when not considering OIST approach and in orange in the other case. One can see that due to the disturbance $d$, the ball is ejected from the beam. When OIST is considered, the ball trajectory remains in the expected interval (in red). Note that the applied saturations result in some conservatism due to the lack of knowledge on $d$ and $e$. Also note that sharper variations are witnessed on the command when applying OIST approach. The trade-off between performance and command energy can be tuned using the parameters $\breve{\kappa}$.

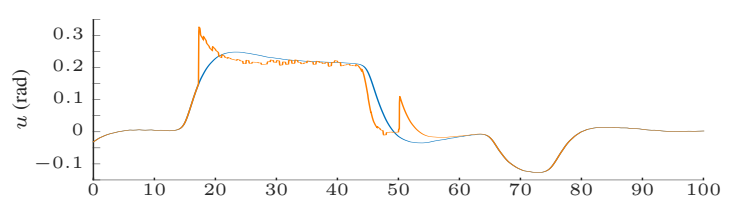

Fig. 8. saturated command in orange

\section{CONCLUSIONS AND FUTURE WORKS}

In this article, the problem of keeping a linear system output - or regulated variable - in an interval has been formalized. A solution based on a transformation from this output expected 'saturation' to a saturation on the available linear control has been proposed. Thanks to the addition of an anti-windup loop, the stability of the system in closedloop with the resulting nonlinear control has been proved. A simple application to a linear system has been proposed.

However, only considering systems where $y=x$ is a bit limited. Future works will be dedicated to extend the approach to systems with matrix $C \neq I_{n}$. On the long term, a synthesis procedure to optimally choose the coefficients $\breve{\kappa}$ could perhaps be imagined. It could also be interesting to study systems with positive zeros for which more complex approaches are required.

\section{REFERENCES}

[1] L. Burlion, "A new Saturation function to convert an output constraint into an input constraint," in Mediterranean Conference on Control and Automation, (Barcelona, Spain), pp. 1218-1222, July 2012.

[2] L. Burlion and H. de Plinval, "Keeping a Ground Point in the Camera Field of View of a Landing UAV," in Proceedings of the IEEE International Conference on Robotics and Automation, pp. 5763-5768, 2013.

[3] M. C. Turner and I. Postlethwaite, "Output violation compensation for systems with output constraints," IEEE TAC, vol. 47, no. 9, pp. 15401546, 2002.

[4] O. J. Rojas and G. C. Goodwin, "A simple anti-windup strategy for state constrained linear control," in Proc. of the 15th IFAC World Congress, (Barcelona, Spain), 2002.

[5] P. P. Menon, G. Herrmann, M. C. Turner, D. G. Bates, and I. Postlethwaite, "General Anti-windup synthesis for input constrained nonlinear systems controlled using nonlinear dynamic inversion," in Proceedings of the 45th IEEE Conference on Decision and Control, (San Diego, CA, USA), pp. 5435-5440, Dec. 2006.

[6] G. Herrmann, P. P. Menon, M. C. Turner, D. G. Bates, and I. Postlethwaite, "Anti-windup synthesis for nonlinear dynamic inversion control schemes," International Journal of Robust and Nonlinear Control, pp. 1465-1482, 2010.

[7] N. Kapoor, A. R. Teel, and P. Daoutidis, "An Anti-Windup Design for Linear Systems with Input Saturation," Automatica, vol. 34, no. 5, pp. 559-574, 1998.

[8] G. Grimm, J. Hatfield, I. Postlethwaite, A. R. Teel, M. C. Turner, and L. Zaccarian, "Antiwindup for Stable Linear Systems with Input Saturation: An LMI-Based Synthesis," IEEE Transactions on Automatic Control, vol. 48, pp. 1509-1525, Sept. 2003.

[9] S. Tarbouriech and M. Turner, "Anti-windup design: an overview of some recent advances and open problems," IET Control Theory and Applications, vol. 3, no. 1, pp. 1-19, 2009.

[10] X. Hu, A. Lindquist, J. Mari, and J. Sand, "Geometric Control Theory." 2012. 